\title{
RDUS
}

Revue de DROIT

UNIVERSITÉ DE SHERBROOKE

Titre : DE L'EXPERTISE JUDICIAIRE DANS LE CADRE DU PROCÈS CRIMINEL ET DE LA RECHERCHE DE LA VÉRITÉ : QUELQUES RÉFLEXIONS

Auteur(s): $\quad$ Pierre PATENAUDE

Revue : $\quad$ RDUS, 1996-1997, volume 27, numéro 1-2

Pages: $\quad 1-47$

ISSN : $\quad 0317-9656$

Éditeur : Université de Sherbrooke. Faculté de droit.

URI : $\quad$ http://hdl.handle.net/11143/12850

DOI : https://doi.org/10.17118/11143/12850 
Page vide laissée intentionnellement. 


\title{
ARTICLE
}

\section{DE L'EXPERTISE JUDICIAIRE DANS LE CADRE DU PROCÈS CRIMINEL ET DE LA RECHERCHE DE LA VÉRITÉ : QUELQUES RÉFLEXIONS}

par Pierre PATENAUDE*

\begin{abstract}

\section{Scientific expertise and the adversarial system}

Lawyers consider science a means of attaining professional ends. They will select or reject experts and evidence according to the needs of their clients.

Indeed, Douglas M. Lucas, then director of the Centre of Forensic Science of Toronto once wrote :

«The scientist must learn, or at least accept, that the judicial process is not a search for truth in the scientific sense, but rather a search for truth as defined by one or the other of the adversaries» ${ }^{1}$
\end{abstract}

So the role of the expert in court is a difficult one because of pressures from the attorney who hires the expert, and because of a strong tendency to identify with the side on whose behalf one is working.

This is disturbing : should not the expert assist the judge in the quest for truth? Should we not protect the autonomy of the scientist so that his research may be done with complete impartiality?

*. $\quad$ Professeur titulaire à la Faculté de droit de l'Université de Sherbrooke. L'auteur tient à remercier spécialement M. André Münch et M. Jean A. Morin, retraités du Laboratoire de sciences judiciaires et de médecine légale du ministère de la Sécurité Publique du Québec pour l'accès aux documents en leur possession et pour leurs commentaires ainsi que le British Council et l'Université de Sherbrooke pour leur appui financier.

1. Douglas M. Lucas, «The Ethical Responsibilities of the Forensic Scientist : Exploring the Limits», (1989) 34 J. Forensic Sci. 719 à la p. 722. 
De l'expertise judiciaire dans le cadre du procès criminel et de la recherche de la vérité : quelques réflexions

Would the Continental legal system be preferable, where the judge appoints experts of his or her choice?

\section{The status of forensic laboratories}

Most forensic laboratories are under direct administration of the police. In Quantico, the F.B.I. national academy is headed by police administrators and staffed with policemen; in Lyon, the Direction centrale de la police judiciaire has an equivalent status. Elsewhere, we may have forensic laboratories with greater autonomy : the Forensic Science Service in Britain and Quebec's Laboratoire de sciences judiciaires et de médecine légale are autonomous agencies; nevertheless they are subject to the general authority of the Home Office or the Ministère de la sécurité publique respectively.

One should not overlook the fact that subjectivity is present even in forensic research. To substantiate this, I look at what is considered as one of the most reliable and objective forms of forensic evidence, namely fingerprinting.

If subjectivity can affect the decision of the fingerprint expert, what does this bode for other forensic sciences such as writing identification, spectographic evidence, psychology, psychiatry or even anthropology where subjectivity has a more important role?

Indeed, we know that organizational philosophy can sometimes affect subjectivity. A forensic scientist who has always worked for the prosecution in a Center devoted to police work, having knowledge of the case in issue, working in a building shared with police administrators, being submitted to a superior who has devoted his professional life to police enquiry, such an expert is in an ideal situation to become biased. We would never accept such a situation for a judge. Yet we know that the forensic expert's evidence is often determinative.

How can we rectify this situation, at least partially? 


\section{Assessing complex scientific evidence}

In Daubert v. Merrell Dow Pharmaceuticals Inc. ${ }^{2}$ the Supreme Court of the United States suggested that judges should use scientific methods to evaluate the value of a science. It has indicated that peer review should be considered very important :

«Widespread acceptance can be an important factor in ruling particular evidence admissible, and a known technique that has been able to attract only minimal support within the community ... may properly be viewed with skepticism».

In that decision, at least utilization of «grille d'analyse», a method, a test, has been imposed upon lower courts to determine admissibility. It has been stated that validity may affect not only weight, but also admissibility.

In Canada, pertinent evidence is normally admissible. Nevertheless a judge may declare inadmissible certain scientific evidence if he is convinced, during voir-dire, that its probative value is weak and that to receive such evidence would be greatly detrimental or cause important prejudice. ${ }^{3}$ Yet the Supreme Court has not established any grille d'analyse to determine the validity and probative value of novel scientific evidence.

Would it not be preferable to have a pre-established grille d'analyse [key of interpretation or method] imposed along with a requirement that it be shown that such method has indeed been followed? In other words would it not be preferable that a formalised system of legally enforceable controls on the admissibility of such expert evidence be put in place?

And shouldn't the judge always be assisted by a court-appointed expert in this task?

2. Daubert v. Merrell Dow Pharmaceuticals, Inc., 113 S. Ct. 2786 at p. 2797 (1993) (cert. denied 116 S. Ct. 189 (1995)).

3. $\quad$ R. v. Mohan, [1994] 2 R.C.S. 9. 
De l'expertise judiciaire dans le cadre du procès criminel et de la recherche de la vérité : quelques réflexions

This expert would not have had the prior instruction to find everything he could to win a client's case. He would, as a general rule, be much more impartial than the parties' experts.

This court-appointed expert would have to submit a written report which would be handed over to both parties; he would be questioned and confronted by them. The judge's attention would be drawn to the questionable parts of the expertise. He would then be able to limit his study to certain specific points. Thus, the search for truth would be better served.

And the advantages of both cross-examination in the adversarial system and the presence of an impartial judge-appointed expert as in the continental system would be present.

\section{Conclusion}

The creation in universities of autonomous forensic laboratories from which prosecution, police, defense and judges could seek assistance, the presence of impartial experts to assist judges in complex scientific litigation, the preservation of the right to cross-examination, the establishment of international committees of certification are some of the recommendations which, we submit, merit consideration. 
SOMMAIRE

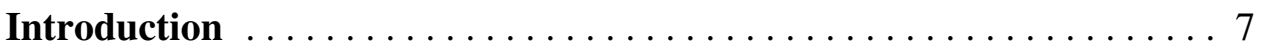

I- De l'indépendance de l'expert dans le cadre du système contradictoire

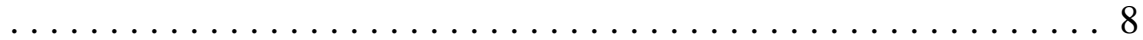

II - De l'évaluation d'une preuve scientifique complexe dans le cadre du

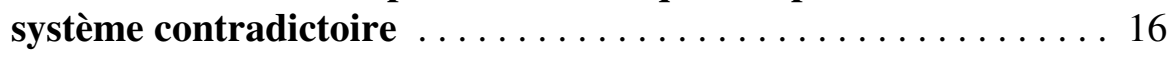

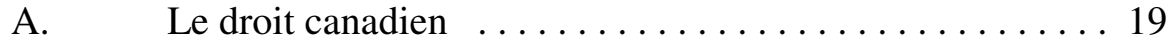

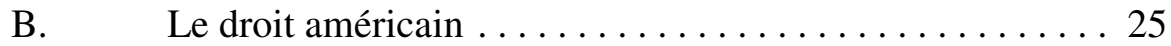

III - De la situation en droit européen continental : l'indépendance de

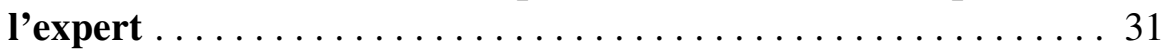

A. La procédure pénale de l'Europe continentale (bref survol) 31

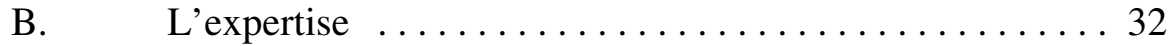

IV- Quelques suggestions pour assurer une meilleure objectivité des experts et une efficience accrue $\ldots \ldots \ldots \ldots \ldots \ldots \ldots \ldots$

V - De l'indépendance institutionnelle des centres d'expertise en crimina-

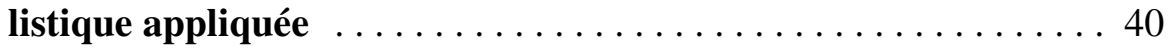

A. L'exemple de la preuve d'empreintes digitales ......442

B. L'indépendance institutionnelle ........... 45

VI- De la création de comités d'agrément ou d'accréditation . . . . 46

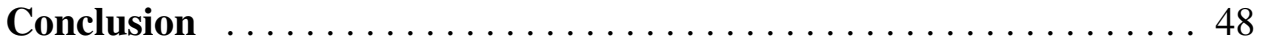





\section{Introduction}

Le climat n'est pas au beau fixe dans les relations entre les experts en criminalistique appliquée (forensic scientists), en sciences judiciaires, tant au niveau civil que pénal, et les juristes, tant avocats que juges. D'ailleurs, plusieurs erreurs judiciaires causées soit par la méprise, soit par l'incom-pétence ou la tromperie des témoins experts ${ }^{1}$ ou encore par l'incompréhension (incompé-

1. Pensons aux cas classiques des Guilford Four et des Birmingham Six en Angleterre, ainsi qu'au cas de l'expert Alan Clyft, en Écosse, expert «forensique» qui dut démissionner après qu'on eut constaté que ses «expertises» erronées avaient amené la condamnation d'innocents; à l'affaire Gregory en France; aux affaires relatives à l'explosion de la Tour de la place World Trade Center et de la mauvaise identification de l'auteur de l'explosion de la bombe d'Atlanta, ainsi que celle d'Oklahoma City, dépendant des résultats de laboratoire (erreurs qui amenèrent un important remaniement de personnel aux laboratoires du FBI); ainsi qu'à l'affaire médiatico-judiciaire O.J. Simpson aux États-Unis, et, plus près de nous, à l'affaire Guy-Paul Morin, en Ontario (expertise de fibres). Au civil, mentionnons l'Affaire Berthiaume c. Val Royal La Salle, [1992] R.J.Q. 76, affaire relative à la mousse isolante d'urée formaldéhyde; un expert avait fondé son témoignage sur des articles dont elle n'avait lu que les résumés apparaissant sur ordinateur. Le juge Hurtubise écrivit d'ailleurs à son sujet : «Se contenter de repérer de la sorte peut dénoter une habileté technique mais certes pas une démarche scientifique sérieuse et achevée, pour parler par euphémisme» (p. 162). et, à propos de cette «experte», il explicite : «Nous déplorons et dénonçons cette attitude irresponsable de la $\mathrm{D}^{\text {re }}$ Sherman. À vrai dire, la cour a perdu confiance en ce témoin qui se refuse ouvertement à accomplir ce devoir d'expert et qui a manifesté au grand jour sa volonté de ne pas éclairer la cour d'une façon honnête et impartiale et de priver la partie adverse de l'exercice de son droit à une défense pleine et entière. Au-delà de l'extrait capital de son rapport que la cour a rayé, ce comportement déteint à nos yeux sur la crédibilité entière du témoin qui nous a semblé davantage un prosélyte qu' un expert impartial» (p. 162) Nous comprenons la récrimination du juge, alors qu'il écrit : «Seul au milieu du champ ... il incombe au juge d'interpréter les témoignages nettement contradictoires que lui ont livrés un très grand nombre d'experts dont certains lui ont semblé hautement préoccupés par le triomphe d'un point de vue ou d'une école de pensée dont ils affichaient le panache» (p. 118). Dans Gburek c. Cohen, [1988] R.J.Q. 2424 (C.A.), monsieur le juge Beauregard avait fait des commentaires dans le même ordre de pensée : «Ces causes de responsabilité médicale posent, outre des questions de droit complexes, des problèmes dont la solution nécessite que le juge ait, sinon des connaissances en médecine, au moins assez de notions scientifiques pour apprécier la force probante relative des témoignages contradictoires des experts. À mon avis, en entreprenant l'étude d'une telle cause, le juge ferait bien pour tout le monde de s'adjoindre un expert indépendant. La longueur de mon délibéré s'explique par la difficulté que j'ai eue à évaluer le témoignage des experts sans l'aide d'un expert indépendant (...) Enfin, même si cela est bien humain, il faut déplorer que certains experts se méprennent sur leur rôle et qu'ils se croient obligés d'épouser la cause de leur client plutôt que de simplement exprimer leur opinion d'une façon objective. Ceci rend 
De l'expertise judiciaire dans le cadre du procès criminel et de la recherche de la vérité : quelques réflexions

tence ... (!)) des juges et avocats face aux principes sous-jacents aux techniques utilisées par les experts pour fonder leurs conclusions, erreurs qui firent la une des médias, discréditèrent grandement tout le système judiciaire et ternirent la réputation tant des juristes, des policiers que des experts.

Mais d'où viennent cette apparente incompatibilité, souvent même ce climat d'antagonisme et de méfiance, qui caractérisent parfois les contacts entre le prétoire et la science?

À notre avis, le système contradictoire est la cause première de cet état de fait. Nous verrons pourquoi. Les juristes sont donc convoqués à une remise en question de ce système, à tout le moins lorsque l'expertise est requise.

Puis, l'indépendance même de l'expert doit être mieux assurée pour garantir son impartialité : indépendance tant au point de vue individuel qu'institutionnel. Et là, c'est toute la structure du travail de l'expert qui est remise en cause.

Sans doute, les forces de l'inertie sont puissantes; la crainte face aux changements est pérenne! Nous savons déjà que modifier les habitudes de travail des professionnels est ardu. Mais la recherche de la vérité, qui doit être le but ultime de la preuve, impose, à notre avis, des changements substantiels.

\section{I- De l'indépendance de l'expert dans le cadre du système contradictoire}

L'indépendance de l'expert est souvent mise en question dans le cadre de notre système contradictoire. Dans une récente décision de la Cour d'appel d'Angleterre, relative à l'expertise d'évaluateurs immobiliers, un extrait campe bien le problème de la difficile coexistence de ce système et de l'expertise en preuve :

évidemment plus nécessaire que le juge nomme son propre expert.» (à la p. 2442) Comme quoi plusieurs des problèmes que nous soulèverons quant à l'expertise dans le cadre du procès criminel pourront être transposés au cas de litige civil! 
«It was argued that appointment of a court expert was pointless, since it merely meant the instruction of an additional expert whose opinion would carry no more weight than any other.

We feel bound to say that in our opinion this argument ignores the experience of the courts over many years. For whatever reason, and whether consciously or unconsciously, the fact is that expert witnesses instructed on behalf of parties to litigation often tend, if called as witnesses at all, to espouse the cause of those instructing them to a greater or lesser extent, on occasion becoming more partisan than the parties. There must be at least a reasonable chance that an expert appointed by the court, with no axe to grind but a clear obligation to make a careful and objective valuation, may prove a reliable source of expert opinion. If so, there must be a reasonable chance at least that such an opinion may lead to settlement of a number of valuation issues.» ${ }^{2}$

La présence de l'expert devant les tribunaux se justifie par la recherche de la vérité; il est clair que le témoignage de l'expert n'est recevable que lorsque ce dernier opine sur un sujet sur lequel juges et jurés sont incompétents; ces constatations furent souvent reprises par la Cour suprême du Canada; limitonsnous à deux extraits pertinents :

«Le rôle d'un expert est précisément de fournir au juge et au jury une conclusion toute faite que ces derniers, en raison de la technicité des faits sont incapables de formuler. L'opinion d'un expert est recevable pour donner à la cour des renseignements scientifiques qui, selon toute vraisemblance, dépassent l'expérience et la connaissance d'un juge ou d'un jury. $\mathrm{Si}$, à partir des faits établis par la preuve, un juge ou un jury peut à lui seul tirer ses propres conclusions, alors l'opinion de l'expert n'est pas nécessaire.» ${ }^{3}$

«Le rôle du témoin expert consiste à mettre à la disposition du jury ou de tout autre juge des faits son opinion d'expert sur le sens de faits établis, ou sur les conclusions à en tirer, dans un domaine où le témoin expert possède des connaissances et une expérience spéciales qui dépassent celles du juge des faits. Il est permis au témoin expert d'exprimer de telles opinions pour

2. Abbey National Mortgages PLC v. Key Surveyors Nationwide Ltd. and others, [1996] 3 All E.R. 184 (C.A.) à la p. 191.

3. R. c. Abbey, [1982] 2 R.C.S. 24 à la p. 42. 
aider le jury. Toutefois, lorsqu'il s'agit d'une question qui relève des connaissances et de l'expérience du juge des faits, point n'est besoin du témoignage d'un expert et, à ce moment-là, aucune opinion d'expert ne sera admise.» ${ }^{4}$

On comprend donc que l'expert est un auxiliaire du juge des faits, il est présent pour combler l'ignorance de ce dernier sur des questions spécifiques qui sont hors de son art. Le juge, donc, conscient de la complexité de l'affaire dont il est saisi et connaissant ses limites ou encore celles des jurés, accepte le témoignage de l'expert, recourt aux lumières des spécialistes, déroge aux règles générales de preuve relatives à l'irrecevabilité de l'opinion, pour privilégier la recherche de la vérité.

La recherche de la vérité est donc la seule justification de la présence de l'expert au prétoire.

Or, pour que cette dernière soit possible, encore faut-il que l'impartialité de l'expert soit protégée et que celui-ci dispose des meilleures conditions de travail. Pourtant, notre système contradictoire (adversarial) répond mal à cet impératif; tout semble mis en oeuvre pour miner l'objectivité et l'impartialité du savant appelé à la barre. ${ }^{5}$

Premièrement, l'expert est celui des parties : il est choisi par le procureur de celles-ci et son témoignage devient une composante importante de sa stratégie : l'expert doit l'aider à gagner sa cause. D'ailleurs, l'avocat veillera à bien préparer le témoignage de «son» expert : il exigera souvent que ce dernier taise certaines nuances qui pourraient s'avérer néfastes à sa cause, qui pourraient amener juge ou jurés à perdre le fil de la structure dialectique de l'argumentation et surtout, le plaideur fera parfois taire le doute! Il ressort d'ailleurs d'une étude

4. La Reine c. Béland et Phillips, [1987] 2 R.C.S. 398 à la p. 415.

5. O. Hilton, Ethics and the Document Examiner Under the Adversary System, [1976] $21 \mathrm{~J}$. Forensic Sci. 779; E. Tanay M.D., Money and the Expert Witness : an Ethical Dilemma, (1976) 21 J. Forensic Sci. 769; J. D. Havard, «Expert Scientific Evidence Under the Adversarial System : A Travesty of Justice», (1992) 32 J. For. Sci. Soc’y. 225. 
américaine, ${ }^{6}$ que quarante pour cent $(40 \%)$ des avocats qui ont répondu au sondage ont mentionné l'indécision, les conclusions nuancées, comme étant l'un des plus grands défauts des experts : souvent le plaideur exigera donc des conclusions péremptoires. ${ }^{7}$

Deuxièmement, il semble bien que certains experts se complaisent dans un rôle partisan qui peut parfois s'avérer fort lucratif. ${ }^{8}$ Cette pratique est devenue tellement courante que plusieurs sociétés professionnelles spécialisées en expertises judiciaires furent contraintes d'adopter des codes d'éthique qui, néanmoins, n'ont aucune force contraignante. ${ }^{9}$

C'est donc à bon escient que le directeur du Centre of forensic science de Toronto écrivait à l'intention des experts en sciences judiciaires :

«The scientist must learn, or at least accept, that the judicial process is not a search for truth in the scientific sense, but rather a search for truth as defined by one or the other of the adversaries. ${ }^{10}$

Évidemment, une telle situation n'est pas propre à améliorer les relations entre avocats et scientifiques. Ces derniers ont souvent une piètre opinion de

6. D.W. Shuman; E. Whitaker; A. Champagne, «An Empirical Examination of the Use of Expert Witnesses in the Court-Part II: A three City Study», (1994) 34 Jurimetrics Journal 193-208.

7. A. Galluser, L'indice matériel comme moyen de preuve: sa valeur et son utilisation par les magistrats, Thèse de doctorat présentée à l'Institut de police scientifique et de criminologie de l’Université de Lausanne, Lausanne, le 10 octobre 1997, 277 p. aux pp. 25 à 48.

8. O. Hilton, supra note 5 à la p. 780.

9. O. Hilton, ibid., «Among forensic scientists who are not members of these established professions, the document examiner may be somethat unique as he does have a written code of ethics ... The fact that the code grew out of this experience is important. The authors had served frequently as expert witnesses employed by litigants eager to gain advantages from this testimony. They had encountered on more than one occasion those of a group of forensic experts who might better be described as forensic science advocates, individuals who endeavor to do the very best they can for their client's case and who in time develop a partial blindness to everything that might seem adverse»; J. L. Peterson \& J. E. Murdock, Forensic Science Ethics Developing an Integrated System of Support and Enforcement, (1989) 34 J. Forensic Sci. 749.

10. D. M. Lucas, «The Ethical Responsibilities of the Forensic Scientist: Exploring the Limits», (1989) 34 J. Forensic Sci. 719 à la p. 722. 
ceux-là, les considérant prêts à tout pour gagner leur cause. Mais que dire maintenant de certains juristes qui affublent les témoins experts de qualificatifs peu flatteurs tels que «saxophones» (signifiant qu'ils jouent le morceau que l'avocat leur commande) ou «hired guns». ${ }^{11}$ Cette soumission du scientifique aux directives du juriste n'est certes pas un incitatif à une participation des meilleurs scientifiques. ${ }^{12}$

«Lawyers frequently consider forensic science a utilitarian tool to further their professional ends. They will select or reject experts and evidence according to the needs of their client ${ }^{13}$. (...) For the scientists hired by counsel as an expert witness, compromises are made in what evidence is solicited from the witness at the discretion of the attorney, and these militate against the scientist autonomy. After all, it is the attorney who attracts the client, orchestrates a defense or prosecution, and allocates the resources available. The attorney manages the entire case, while the expert witness is one among many role players. ${ }^{14}$

«The adversary system casts the expert witness in a partisan role. Counsel tries to extract a slanted picture from the witness, and, on crossexamination, opposing counsel seeks to slant the picture the other way. To the man trained in objectivity, this is a perversion of a quest for truth and justice. That some of his colleagues have adapted successfully to its pressure makes the situation all the more obnoxious to him.» ${ }^{15}$

Cette opinion est corroborée par plusieurs écrits provenant des experts en criminalistique. ${ }^{16}$ En voici une qui ne laisse pas place à l'interprétation :

11. Expressions tirées de l'article du doyen J. R. Spencer, «Court Experts and Expert Witnesses: Have we a Lesson to Learn from the French?», (1992) 45 Current Legal Problems, 211 à la p. 220.

12. Ibid.à la p. 223. Voir aussi supra note 6.

13. M. S. Frankel, «Ethics and the Forensic Sciences: Professional Autonomy in the Criminal Justice System», (1989) 34 J. Forensic Sci. 763 à la p. 764.

14. Ibid. à la p. 767.

15. D.V. Cavers, dans Law and the Social Role of Science, par H.W. Jones, ed. Rockefeller University Press, New York, 1966, p. 6.

16. D. M. Lucas, supra note 10 à la p. 723. «The other-sidedness thought processes of the adversary system can have significant detrimental effects on the desire for impartiality of the scientist, "gamesmanship" is a legitimate function of police and attornies, and the temptation for the scientist to enter the game is subtle but strong»; J. L. Peterson \& J E. Murdock, supra 
«... in the atmosphere of courtroom advocacy. There is the ever-present danger of partiality of unconsciously becoming biased if one is not careful, regardless of whether one works in the public service continuously with the investigation of crime and as a witness for the prosecution, or as a consultant in criminal matters dealing primarily with the defense. In other words, being a frequent part of one or another team of advocates can color one's approach to a problem ... Thus, one may become less objective and impartial. Accuracy and the thoroughness may suffer. ${ }^{17}$ (...) These men, because of constant work with either prosecution or defense, have lost touch with the fact that all written and oral reports should be technically correct and conservative and strictly in accordance with the physical evidence. There are certain law enforcement examiners who almost never exonerate a suspect, and various defense-oriented experts who fail to report positive identifications of any defendants. ${ }^{18}$

Pouvons-nous, sans sourire, affirmer que ce commentaire relatif à l'expert en écriture n'est pas pertinent à plusieurs autres domaines d'expertise? Pensons, entre autres choses à certaines expertises psychologiques et psychiatriques ...!

Et que dire du contre-interrogatoire souvent marqué du sceau de l'agressivité. Est-il vraiment le moyen privilégié pour que la vérité scientifique éclate?

«Unless he is a rogue or a madman, the most truth-producing way to challenge a scientist is to get him to discuss the matter calmly with another scientist who holds the opposite view, and to give him a chance to reflect quietly on his colleague's opinions before he replies. By contrast, waiting until the scientist has publicly committed himself to a particular view and then making him answer questions off the cuff, under stress, and at a public hearing, is an inefficient method. It makes the stubborn ones dig their heels in when they should concede, and the ones who cannot think on their feet concede points when, given time to reflect upon them, they would see the points were bad.» ${ }^{19}$

note 9.

17. O. Hilton, supra note 5 p. 779.

18. Ibid. à la p. 781.

19. Supra note 11 à la p. 223. 
Le biochimiste Léo Lavergne du Laboratoire de sciences judiciaires et de médecine légale au ministère de la Sécurité publique de Montréal, en témoignant de son expérience en tant que témoin expert en identification génétique, écrit :

«Dans un autre ordre d'idées, la partie adverse pourrait utiliser une stratégie de confusion qui, par son approche complexe, tentera de faire perdre confiance aux membres du jury en exposant l'ignorance de ces derniers et leur incapacité à juger de faits hautement techniques. Dans une telle situation, l'avocat complexifie ses questions, utilise plus de termes scientifiques et du même coup détruit l'ambiance plus simple et sécurisante qu'avait créée le spécialiste lors de son interrogatoire principal. Pour contrer une riposte de ce genre, le témoin expert devra ramener la terminologie des questions en un vocabulaire accessible au jury, expliquer les nouveaux termes que ces derniers ont entendus, faire le rapprochement avec le témoignage principal s'il y a lieu et, le cas échéant, positionner ces nouveaux concepts dans la problématique globale du message à livrer en répondant à la question. De cette manière, le témoin expert conserve le contrôle sur la valeur de son message, gagne en crédibilité devant le jury qui comprend à nouveau ce spécialiste qui sait s'exprimer et se faire comprendre tout en désamorçant la tactique de l'adversaire. Il va sans dire que toute cette stratégie de communication ne sera vraiment efficace qu'avec des compléments essentiels tels une attitude polie, un débit de voix assez lent permettant à l'interlocuteur de saisir toutes les nuances du discours et un contrôle de ses émotions assorti d'une patience exemplaire. ${ }^{20}$

Toutes ces constatations sont justes et fort pertinentes dans le cadre de notre système contradictoire. L'expert, pour que son témoignage porte fruits doit préférablement être un communicateur, un vulgarisateur, toujours conserver un débit de voix assez lent et un contrôle de ses émotions, et ce, dans une atmosphère souvent survoltée alors qu'il est la victime d'un avocat parfois agressif le tourmentant de questions évidemment sournoises dont les réponses

20. L. Lavergne, «Deux aspects de l'identification génétique en médecine légale : la position du généticien dans ce nouvel environnement et l'évaluation de rareté des profils génétiques» dans C. Hennau-Hublet et B. M. Knoppers, L'Analyse génétique à des fins de preuve et les droits de l'homme, Bruylant, Bruxelles, 1997, 509 p. aux pp. 46-47. 
pourraient avoir des conséquences importantes sur l'issue du procès ainsi que sur sa propre réputation. Et de plus, l'expert se voit investi de la tâche de «désamorcer la tactique de l'adversaire».

Mais, si ces qualités sont essentielles au témoin expert, et nous ne doutons pas qu'elles le soient, sont-elles propres à attirer au prétoire les meilleurs savants? Combien d'experts, qui ont passé une longue partie de leur vie en laboratoire, sont vulgarisateurs, éducateurs, psychologues, doués d'un contrôle de leurs émotions? Ne se pourrait-il pas que celui qui saurait présenter la vérité scientifique soit, dans certains cas, un «ours mal léché», allergique au public, souvent importuné d'avoir été retiré de son laboratoire pour s'exhiber au prétoire; qu'il ne puisse quitter son vocabulaire hermétique au profit de celui du peuple? Bref, ce savant, sachant ce qui l'attend, préférera parfois s'abstenir, même si la justice et la vérité risquent d'en pâtir... à moins que le système ne soit modifié pour rendre le prétoire plus accueillant et plus adapté à la science, ce que nous verrons plus loin.

Bref, le système contradictoire est loin d'assurer la qualité, l'indépendance et l'impartialité de l'expert; enfin, nous le verrons, il n'accorde pas aux juges des faits les moyens d'exercer efficacement leur tâche; même si, en théorie, le juge au procès pourrait s'adjoindre un expert de la cour, car ce privilège est, en pratique, fort peu exercé.

Quelques protections minimales contre l'erreur existent, néanmoins, en droit canadien et américain lors de présentation d'une preuve scientifique complexe ou non validée, ce que nous verrons maintenant.

\section{II - De l'évaluation d'une preuve scientifique complexe dans le cadre du système contradictoire}

Nous avons déjà eu l'occasion de souligner jusqu'à quel point notre système juridique est inapproprié lorsqu'il confie à un juge la tâche d'évaluer 
une preuve scientifique complexe. ${ }^{21}$ On peut comprendre le désarroi des juristes quand la science fait son entrée au prétoire. Nous écrivions en 1992 :

«Déjà le témoignage fondé sur une expertise traditionnelle peut parfois être source de perplexité chez un non initié aux sciences qualifiées, souvent à tort, d'exactes; l'embarras risque d'être encore plus grand lorsque des témoignages contradictoires d'experts aux propos hermétiques sont présentés; enfin, la confusion la plus totale peut résulter de l'apparition en cour de preuves obtenues grâce au recours à des techniques tout à fait innovatrices dont la qualité et la fiabilité n'ont pas encore été consacrées ni par un consensus de la communauté scientifique concernée, ni par des décisions judiciaires.» ${ }^{22}$

Lorsqu'arrivent en preuve les fruits de l'utilisation d'une technique tout à fait innovatrice ou encore terriblement complexe et lorsque le juge est confronté à des témoignages d'experts tout à fait contradictoires quant à la validité de ladite technique, il doit trancher. Or, à notre avis, sauf dans de très rares exceptions, il n'en a pas la capacité. Son degré de compréhension n'est pas suffisant, lorsque sa seule formation se résume à des études en sciences humaines et à des années de pratique en droit. Alors, le juge se trouve dans la même situation que les théologiens du Moyen-Âge, qui devaient faire le partage entre deux théories scientifiques opposées. Inutile de mentionner que la justice peut parfois en souffrir...(!)

Le doyen de la Faculté de droit de Cambridge, M. le professeur J.R. Spencer, a déjà écrit, qu'à la limite :

«... the court, having no expert knowledge of its own, is ill-placed to decide between the evidence that it has heard - with the result that the battle of the experts degenerates into a simple beauty contest.» ${ }^{23}$

21. P. Patenaude, «La preuve, les techniques modernes et le respect des valeurs fondamentales», Éd. Revue de droit Université de Sherbrooke, 1990, 296 p. aux pp. 145 et ss. P. Patenaude, «Sciences et techniques en preuve : nécessaire réforme du droit civil, indispensable adaptation du système et des acteurs», dans Développements récents en droit civil (1992), Service de la formation permanente, Barreau du Québec, Éd. Yvon Blais, Cowansville, 1992, p. 77-100.

22. P. Patenaude, «Sciences et techniques en preuve...», ibid. aux pp. 93-94.

23. Supra note 11 à la p. 224. 
Une telle situation est néfaste en droit civil, ${ }^{24}$ mais elle est encore plus inacceptable en droit criminel, car il peut se présenter des cas où un accusé sera condamné sur la foi d'une expertise mal fondée, de l'utilisation de techniques douteuses.

En outre, lorsque la poursuite fonde sa preuve en partie sur les résultats de l'utilisation d'une technique tout à fait nouvelle, la défense est souvent fortement défavorisée : alors que les dépenses inhérentes à la poursuite émargent au budget de l'État, le prévenu doit souvent assumer lui-même les coûts de la défense. Lorsque la preuve repose sur des éléments obtenus au moyen de l'utilisation de techniques conventionnelles (par exemple, le radar, l'alcootest, les radiographies..., etc.), il est normalement facile de trouver localement des experts pour présenter une contre-expertise. Mais si des moyens techniques innovateurs, nouveaux, ont produit la preuve, la poursuite peut, aux frais du gouvernement, appeler à la barre des témoins le concepteur pour qu'il établisse la validité du principe fondamental, la fiabilité des appareils utilisés ainsi que la compétence des techniciens qui les ont opérés. En revanche, dans certains cas, la défense ne pourra pas faire venir un expert-opposant qui aurait pu présenter une contre-expertise lorsque celui-ci oeuvre dans un endroit fort éloigné du lieu du procès, les finances du défendeur ne permettant pas d'assumer les frais de déplacement et les honoraires du spécialiste.

Dans le cadre d'un rapport d'une commission royale d'enquête australienne, un juge écrivait :

«In many cases, the opinions expressed by the Crown's experts are accepted by the defence. If they are not accepted, the resources of the accused person may well not suffice to enable him to challenge them. $»^{25}$

La poursuite bénéficie, en outre, des nombreuses ressources des laboratoires de police scientifique, nommés aussi laboratoires de criminalistique

24. P. Patenaude, «Sciences et techniques en preuve : nécessaire réforme du droit civil, indispensable adaptation du système et des acteurs», supra note 21.

25. J.R. Morling, Report of the Royal Commission of Inquiry into the Chamberlain Convictions, Government Printer, Darwin, Northern Territory, Australia, 1987, rapporté dans D. M. Lucas, supra note 10 à la p. 721. 
appliquée; les spécialistes de ces instituts sont nombreux, bien équipés et ont des contacts avec les laboratoires de sciences «forensiques» partout dans le monde. Leurs services aux corps policiers et à la poursuite sont gratuits, en ce sens que l'État prend à sa charge les frais de ces centres.

À l'opposé, l'avocat de la défense peut certes avoir recours à des experts indépendants ou à des spécialistes oeuvrant dans les universités; mais aura-t-il, localement, le savant apte à présenter une contre-expertise? Sinon, connaîtra-t-il l'existence d'un spécialiste étranger qui le pourrait? À notre avis, l'État devrait prévoir une aide financière pour assurer à l'accusé l'accès à une telle contreexpertise.

Prenant en considération le caractère souvent déterminant de l'expertise et son effet d'ordinaire péremptoire sur la décision, donc, considérant les dangers d'une expertise mal fondée, M. le professeur Peter Alldridge recommande que l'expertise présentée par la poursuite soit soumise, en voirdire, à un test analogue à celui imposé lors de confessions extrajudiciaires faites à une personne en autorité :

«One method of generating safeguards would be to provide that the evidence should only be admitted - as in the case of confessions - in the case where the prosecution is able to establish beyond reasonable doubt that each of the appropriate procedures have been adopted.» ${ }^{26}$

Mais, même dans une telle hypothèse, celui qui aurait à s'assurer de la qualité du travail de l'expert et de la validité du principe sous-jacent à la technique utilisée serait une personne - le juge - totalement ignorante de la science en cause. Nous ferons valoir qu'il serait préférable que ce dernier s'adjoigne obligatoirement un expert de la Cour, pour l'assister dans cette tâche d'évaluation ou mieux, à notre avis, que l'expertise soit a priori effectuée par un expert de la Cour, choisi par le juge, comme c'est le cas en Europe continentale.

26. P. Alldridge, «Recognizing Novel Scientific Techniques: DNA as a test case», (1992) Crim. L. Rev. 687 à la p. 698. 
Mais voyons auparavant quelle protection accordent les droits canadien et américain à l'accusé, lorsque la poursuite présente les résultats de l'utilisation d'une technique connue depuis peu.

\section{A. Le droit canadien}

Au Canada, le juge se réserve le droit d'exclure une preuve fondée sur une technique non validée si sa valeur probante est surpassée par son effet préjudiciable.

Dans la décision rendue dans l'affaire La Reine c. Mohan, ${ }^{27}$ relative à l'admissibilité d'une preuve d'expert concernant le profil psychologique d'un accusé, le juge Sopinka, au nom de la Cour suprême, établira la règle :

«La pertinence est déterminée par le juge comme question de droit. Bien que la preuve soit admissible à première vue si elle est à ce point liée au fait concerné qu'elle tend à l'établir, l'analyse ne se termine pas là. Cela établit seulement la pertinence logique de la preuve. D'autres considérations influent également sur la décision relative à l'admissibilité. Cet examen supplémentaire peut être décrit comme une analyse du coût et des bénéfices, à savoir «si la valeur en vaut le coût.» Le coût, dans ce contexte, n'est pas utilisé dans le sens économique traditionnel du terme, mais plutôt par rapport à son impact sur le procès. La preuve qui est par ailleurs logiquement pertinente peut être exclue sur ce fondement si sa valeur probante est surpassée par son effet préjudiciable, si elle exige un temps excessivement long qui est sans commune mesure avec sa valeur, ou si elle peut induire en erreur en ce sens que son effet sur le juge des faits, en particulier le jury, est disproportionné par rapport à sa fiabilité. Bien qu'elle ait été fréquemment considérée comme un aspect de la pertinence juridique, l'exclusion d'une preuve logiquement pertinente, pour ces raisons, devrait être considérée comme une règle générale d'exclusion. ${ }^{28}$

27. [1994] 2 R.C.S. 9; Commentaires : P. B. Limpert, «Beyond the Rule in Mohan: A New Model for Assessing the Reliability of Scientific Evidence», (1996) 54 U. of T. Fac. Law Review 65; Commentaires : R.J. Delisle, «The Admissibility of Expert Evidence : A New Caution Based on General Principles», (1994) 29 C.R. ( $\left.4^{\text {th }}\right) 267$.

28. Ibid. aux pp. 20-21. (Nos italiques) 
Donc, le juge peut, dans certains cas, déclarer irrecevable le rapport des résultats de l'utilisation d'une technique ou d'une analyse dite scientifique lorsque la validité du principe fondamental n'est pas établie, que la valeur de la technique utilisée est incertaine, douteuse, et à la condition de lui prouver qu'admettre une telle preuve aurait un impact préjudiciable, surtout si son effet est dommageable à l'accusé, ou encore que sa présentation pourrait induire le juge des faits en erreur, parce que son effet sur lui serait disproportionné par rapport à sa fiabilité.

On comprend, à la lecture de cet extrait, que l'analyse de la valeur probante de cette nouvelle technique ou science devra, en premier lieu, être faite dans le cadre de l'étude préliminaire relative à sa réception ou à son rejet.

Si la preuve en est une présentée par la Couronne, la défense alléguera que, vu la non-consécration de cette nouvelle technique par la communauté scientifique pertinente, sa force probante est faible et que sa réception en preuve risquerait d'influencer indûment le jury ou encore de l'induire en erreur (car ce dernier ne dispose pas des moyens lui permettant d'avoir une approche critique), ce qui risquerait d'avoir un effet très préjudiciable sur l'accusé.

En effet, admettre, à l'appui d'une condamnation, à titre d'expertise, l'opinion d'une personne fondée sur une théorie reposant sur des bases fragiles pourrait, dans certains cas, être fortement préjudiciable à l'accusé, puisque le jury ne peut ordinairement avoir une approche critique face à un scientifique ou à quelqu'un qui se targue de l'être.

Ainsi, dans R. c. Nielsen, ${ }^{29}$ la Cour d'appel du Manitoba souleva ce danger d'admettre des opinions scientifiques fondées sur des principes non validés et ce, particulièrement lorsqu'elles sont présentées par la Couronne. En l'instance, une théorie de l'unicité des empreintes de pieds aurait pu être présentée par un témoin expert. Fort heureusement, elle ne s'aventura pas dans ce domaine, et la Cour émit l'opinion que si elle l'eut fait, cette expertise aurait pu être déclarée irrecevable. On reçut donc le témoignage de l'experte anthropologue en comparaison d'empreintes de pieds, mais on souleva alors que, si elle avait 
soulevé sa théorie relative à l'unicité desdites empreintes, on aurait déclaré cette partie du témoignage irrecevable. ${ }^{30}$

Si la preuve en est une présentée par la défense, la Couronne tentera d'établir que son admission pourrait induire en erreur en ce sens que son effet sur le juge des faits serait disproportionné par rapport à sa fiabilité.

Ainsi, dans Corporation professionnelle des médecins c. Bortz ${ }^{31}$ le juge accorda (malheureusement à notre avis) le statut d'expert à un parapsychologue présenté par la défense, mais il jugea néanmoins ses conclusions irrecevables aux motifs suivants :

«Les recherches portant sur la médiumnité conservent une valeur scientifique, mais sans qu'on soit parvenu à en obtenir une accréditation universelle. (...) Il n'entre pas dans les prérogatives du tribunal de consacrer judiciairement l'existence d'une telle science, de sorte que le tribunal ne peut accueillir un tel moyen de défense.... ${ }^{32}$

30. Ibid. aux pp. 68 et 69. [Nos italiques]. «In the process of giving voir-dire evidence Dr. Robbins expressed the view flowing from her studies, that each individual has a unique footprint; that a combination of factors consisting of measurements and contours will be sufficient to provide absolute identification. Whether this view is scientifically correct is open to question. There is simply not enough data and not enough scientific study of that data to establish the validity of Dr. Robbin's opinion. There are grave dangers in allowing evidence to be tendered under the rubric of scientific opinion when its authenticity is still moot ...» «... Dr. Robbins did come before the court as a genuine expert in terms of drawing comparisons between different prints and noting similarities. She demonstrated how measurements could be made and what measurements are significant. She observed the oddities of shape and the contours of markings which will assist in an indentification. (...) Expertise ... is invaluable in a multitude of technical procedures, including the measurement of footprints and the identification of similarities in shape. So long as Dr. Robbins confined herself to the area of technical expertise without the gloss of a scientific theory around it, then her testimony was wholly admissible. In fact, such was the nature of her testimony. When she testified before the jury, she described the measurements she had taken to compare the footprints made by Nielsen in paint on brown paper with the footprints etched in blood. She described unique features or characteristics which appeared in both prints, but she shied away from advancing a scientific theory that the footprints of no two human beings could be the same».

31. [1987] R.J.Q. 2675.

32. Ibid. 
En l'instance, Bortz avait été poursuivi pour pratique illégale de la médecine. Il soutenait, en défense, qu'il n'était pas l'auteur de l'intention reprochée, puisqu'il était sous l'influence des êtres d'un autre monde, qu'étant en transes hypnotiques, il n'agissait que comme un médium et ne servait que comme intermédiaire entre son «patient» et des êtres d'un autre monde. Son avocat tenta de présenter une preuve qu'il qualifiait de «scientifique» fondée sur une «science» occulte définie comme étant le phénomène de la médiumnité. Malheureusement, à notre avis, le juge accepta d'accorder le statut d'expert à un parapsychologue, (!) mais fort judicieusement, selon nous, il refusa d'admettre son témoignage.

En droit canadien, la décision rendue dans l'affaire Mohan a, à tout le moins, réitéré la règle à l'effet que le juge peut rejeter une preuve dont la réception causerait un préjudice disproportionné par rapport à sa force probante. Mais, cette décision fut silencieuse quant aux normes à suivre pour décider de la validité d'une technique auréolée, à tort ou à raison, du qualificatif de scientifique. La Cour jugea que le juge du procès avait eu raison d'exclure l'opinion de l'expert à l'effet que l'accusé ne possédait pas certaines caractéristiques distinctives propres aux individus ayant une propension à commettre le crime imputé en l'instance :

«Les profils de groupe décrits par l'expert n'ont pas été considérés suffisamment fiables pour être utiles. En l'absence d'indices de fiabilité, on ne pouvait pas dire que la preuve serait nécessaire au sens où elle clarifierait utilement une question qui serait autrement inaccessible, ou que la valeur qu'elle pourrait avoir ne serait pas surpassée par la possibilité qu'elle induise le jury en erreur ou le détourne de ses tâches. Compte tenu de ces conclusions... je dois conclure que le juge du procès a conclu à juste titre que, du point de vue juridique, la preuve était inadmissible.» ${ }^{33}$

Mais sur quoi se fonder pour établir le degré de fiabilité d'une nouvelle science ou d'une technique innovatrice? Il y a peu d'indices à ce sujet en droit canadien. Nous espérons que le droit canadien s'alignera sur la décision de la Cour suprême des États-Unis rendue dans l'affaire Daubert. ${ }^{34}$ Mentionnons,

33. Supra note 27 à la p. 38. (Nos italiques)

34. Voir plus loin l'étude du droit américain. 
avant de terminer, qu'une décision australienne ${ }^{35}$ en est venue à des conclusions identiques à celles adoptées par les tribunaux canadiens. Dans cette cause, alors que la Couronne avait présenté une identification par A.D.N. à propos de laquelle il y avait eu un débat entre spécialistes de renom, le juge opta pour l'irrecevabilité au motif que les jurés n'auraient pu choisir entre les théories scientifiques avancées. ${ }^{36}$

35. Van Hung Tran, (1990) 50 A. Crim. R. 233.

36. Ibid. pp. 241-242-243. «Here, of course, we have the added problem that there is only one result out of four, that it cannot be duplicated and that the accused, of course, is deprived of the opportunity of determining whether the test was accurate and there is no other material, as in Elliott's case, to corroborate the findings and it is my view that a jury would not be in a position to determine initially whether there was an upper band or not present. Hunt J., in the same decision said this : "If scientific testing in the particular case is unreliable or if it has a tendency to produce a misleading or confusing impression for the jury, or if the weight to be afforded in the results is so minimal as to preclude the jury being satisfied beyond reasonable doubt, that the Crown has established the fact which it seeks to prove then clearly I have a duty to exclude it from the jury - whether it is a result of ruling that the evidence is inadmissible or whether it is excluded in the exercice of my discretion." In this case certainly it appears to me that the jury could determine whether the lower faint band is a band, but that of itself, as I understand it, would not be adequate data to link the accused with the deceased. Indeed Dr Easteal propounded the view that not only would it not link him but would positively exclude him. Further, there is the added problem of the database from which this material is taken and the question of whether the assumptions, not proven by evidence, are correct assumptions in the circumstances. No-one can really answer that question and that is a further matter that makes these tests unreliable. Whilst I do not wish to be critical of anyone, as I know these matters are very difficult, I believe that because of the views about the presence or otherwise of the upper faint band, and the criticisms of the scientific testing, that to put this evidence before the jury, in my view, would have a tendency to produce a misleading and confusing impression for the jury. Indeed, as I have stated, I am of the opinion that the jury would not be in a position, even given the wide discretion given to juries, to determine the issues and in determining this issue I believe they would be speculating. In any event, even if I were of the opinion that it would be open to the jury to conclude that these were matching bands and they matched the bands of the accused, thereby linking him against a one in 152 coincidental chance, if one accepts that figure, or alternatively one in 87 the state of the evidence is in an unsatisfactory state because of the fact that there is no database for Vietnamese. The question of the difficulties of this sort of evidence has been referred to in the case of Lewis (1987) 29 A. Crim. R. 267, and in particular by Maurice J., as he then was (at 271), where he said this : "Forensic evidence, especially if it goes to a vital issue implicating an accused person in the commission of an offence, may often have a prejudicial effect on the minds of a jury which far outweights its probative value. The jury, being people without scientific training, may often be impressed by an expert's qualifications, appointments and experience and the confident manner in which he expresses his opinion. And yet it ought not be left to such matters alone to provide 


\section{B. Le droit américain}

Les tribunaux américains se sont, quant à eux, posé très souvent la question du rôle des tribunaux face à une preuve scientifique complexe et innovatrice, ce qui a créé une jurisprudence abondante : les opinions y sont très partagées quant aux préalables à l'admission d'une telle preuve scientifique. ${ }^{37}$ Depuis 1923, on imposait la preuve d'un consensus de la communauté scientifique concernée relativement à la valeur du procédé avant d'admettre en preuve les résultats d'une technique révolutionnaire. Cette règle avait été élaborée dans l'affaire Frye c. U.S., une cause relative à l'admissibilité des résultats obtenus au moyen du polygraphe. ${ }^{38}$

Ayant à juger du degré de crédibilité à lui accorder, le tribunal avait élaboré la règle qui a prévalu jusqu'à tout récemment :

«Il est difficile de préciser à quel moment une théorie ou une découverte scientifique passe de l'expérimentation à la démonstration. À l'intérieur de cette zone grise, il faut reconnaître la force probante de la théorie, et bien que les tribunaux acceptent d'emblée les témoignages d'experts basés sur une théorie ou une découverte scientifique reconnue, il faut que ce

a foundation for the jury making an assessment of the probative value of forensic evidence, particularly where there are conflicts in expert testimony, or where it is acknowledged that other experts of more or less equal distinction are unlikely to agree." I think those remarks are very apposite to the problem that confronts me in this case. In any event, if I am wrong in concluding that the jury would not be capable of determining the threshhold question that they would have to determine in this case, I would have exercised my discretion to exclude the evidence, bearing in mind that the weight that could be given to that evidence by the jury would, I believe, be outweighed by the corresponding, prejudicial influence which would be on the minds of the jury and which would be out of all proportion to its true evidential value and that, in the circumstances, I would exercice my discretion to exclude the evidence on that basis.»

37. Au sujet du débat relatif aux conditions de recevabilité de la preuve scientifique innovatrice, voir : Symposium on Science and the Rules of Evidence, (1983) 99 F.R.D. 187.

38. $\quad$ Frye c. U.S., 293 F. 1013 (D.C. Cir. 1923) 
fondement scientifique soit suffisamment prouvé pour être accepté de tous dans le domaine particulier en cause.» ${ }^{39}$

Cette traduction offerte par la Commission de réforme du droit du Canada peut prêter à confusion, le texte original n'indiquant pas que le fondement scientifique devait être accepté de tous, mais bien avoir atteint «a general acceptance» ce qui laissait place à certaines dissidences parmi les scientifiques. L'unanimité n'était donc pas requise, contrairement à ce que semble indiquer la traduction. Constatant que la communauté scientifique n'avait pas reconnu la technique utilisée, le tribunal avait opté pour l'irrecevabilité de ces résultats.

Le temps semble lui avoir donné raison : il appert, en effet, que la technique utilisée par l'expert dans l'affaire Frye (blood pressure detection test) est aujourd'hui considérée non fiable. Les polygraphistes affirment maintenant que leur instrument ne ressemble aucunement à celui alors employé. ${ }^{40}$ Mais si l'expert avait été alors admis à présenter ses conclusions, le jury aurait-il été apte à découvrir les limites de la technique utilisée en l'instance?

Rapidement, certaines oppositions se sont manifestées face à la rigueur des exigences imposées par le jugement rendu dans l'affaire Frye ${ }^{41}$ : ainsi, dans l'affaire U.S. c. Stifel, ${ }^{42}$ la Cour fédérale d'appel décida que la reconnaissance scientifique n'était pas une question de recevabilité, mais une de crédibilité laissée à l'appréciation du jury.

Elle devait alors décider de la recevabilité de l'analyse par activation de neutrons. Il s'agissait d'une cause de meurtre; un jeune homme avait été tué par une bombe envoyée par courrier. À l'aide de l'analyse par activation

39. Traduction : Commission de réforme du droit du Canada, Document de travail 34, Les méthodes d'investigation scientifiques, ministère des Approvisionnements et Services Canada, 1984, 186 p. à la p. 27.

40. S. A. Saltzburg, «Frye and Alternatives» dans Symposium on Science and Rules of Evidence, 99 F.R.D. 187 à la p. 208.

41. P. C. Giannelli, «The Admissibility of Novel Scientific Evidence: Frye v. United States, a Half-Century Later», (1980) 80-2 Colum. L. Rev. 1197. M. McCormick, «Scientific Evidence: Defining a New Approach to Admissibility», (1982) 67 Iowa L.R. 879 à la p. 910.

42. $\quad 433$ F. $2 d 431$ (1970). 
neutronique, l'expert avait établi que les composantes du colis contenant la bombe (carton - ruban adhésif - métal et étiquettes gommées) provenaient du manufacturier des mêmes biens que ceux stockés dans une réserve à laquelle l'accusé avait eu accès.

Aucune objection n'avait été faite quant à l'admission des examens microscopiques ou du test d'absorption atomique, puisqu'il semblait que ces deux méthodes avaient alors acquis leurs lettres de créance de la communauté scientifique. Cependant, l'appelant avait contesté la présentation de l'analyse par activation de neutrons parce qu'étant, à l'époque, une science innovatrice dont la fiabilité n'avait pas été reconnue par la communauté scientifique. ${ }^{43}$

L'appelant avait produit en première instance trois experts qui avaient témoigné à l'effet que cette science était trop nouvelle pour être jugée fiable : malgré cela, le juge avait accepté la présentation de cette preuve. En appel, la Cour opina dans le même sens : elle jugea que la technique par activation de neutrons répondait aux exigences élaborées par le jugement rendu dans Frye, ${ }^{44}$ de plus, la Cour établit que le degré de reconnaissance scientifique n'était pas une question d'admissibilité, mais une de crédibilité devant être laissée au jury :

«Appellant's witnesses criticism of Scott's test methods were fully developed before the jury and were appropriate for that body's consideration. Such rebuttal evidence went to the weight of Scott's testimony - not to its admissibility.

Further, neither newness nor lack of absolute certainty in a test suffices to render it inadmissible in court. Every useful new development must have its first day in court. And court records are full of the conflicting opinions of doctors, engineers and accountants, to name just a few of the legions of expert witnesses.» ${ }^{45}$

43. Watkins and Watkins, «Identification of Substances by Neutron Activation Analysis», 15 Am. Jur. «Proof of Facts» 115, 116-119, (1964) Supplement (1969) 12.

44. U.S. c. Stifel, 433 F. 2d 431, 441 (1970); Frye c. U.S., 293 F. 1013 (D.C. Cir. 1923).

45. Frye, ibid. à la p. 438. [Nos italiques]. 
Notons, de nouveau, que l'emploi de l'expression «absolute certainty» risque d'induire en erreur : ce n'est pas la certitude absolue qui devait, selon Frye, être prouvée avant de permettre la présentation des résultats d'innovations scientifiques, ce qui, en matière scientifique est ordinairement impossible; il suffisait de s'assurer a priori que la fiabilité de cette technique nouvelle avait été reconnue par consensus de la communauté scientifique concernée.

On constate donc que la règle à l'effet de s'assurer a priori d'un certain consensus quant à la valeur du fondement scientifique sur lequel se fonde une expertise ne faisait pas l'unanimité.

Soixante-dix ans après la décision rendue dans l'affaire Frye, la Cour suprême des États-Unis eut à décider, dans une affaire Daubert v. Merrell Dow Pharmaceuticals inc., ${ }^{46}$ si la norme à titre de condition préalable à la recevabilité devait demeurer la preuve du consensus de la communauté scientifique ou plutôt s'il ne s'agissait pas simplement d'un élément affectant la force probante des résultats de la technique. De plus, qui validerait ladite «science»: la communauté scientifique pertinente ou le pouvoir judiciaire? Si la communauté scientifique devait décider, ce serait l'évaluation par les pairs qui deviendrait déterminante. Sinon, ce seraient les juges qui auraient à séparer l'ivraie du bon grain.

La Cour établit alors clairement le pouvoir des juges d'évaluer lesdites «sciences» et de les passer au crible. Elle refusa que l'évaluation des pairs et le consensus de la communauté scientifique soient les seules balises à prendre en considération; néanmoins, la Cour incita les juges à évaluer la «science» contestée selon les méthodes propres aux scientifiques. La majorité élabora, à cet effet, quatre paramètres pour établir la validité de la «science» et la pertinence du témoignage de l'expert :

(1) voir si la théorie et la technique à la base de la preuve présentée ont été mises à l'essai, expérimentées et si elles sont trompeuses ou crédibles; ${ }^{47}$

46. $\quad 113$ S.Ct. $2786(1993)$.

47. Ibid. à la p. 2796. 
(2) s'assurer qu'il y a eu évaluation par les pairs; qu'il y a eu publication relative à cette nouvelle «science»; ${ }^{48}$

(3) prendre connaissance du taux d'erreurs inhérent à ladite technique ou «science»; ${ }^{49}$

(4) voir s'il y a un certain consensus de la communauté scientifique quant à la valeur de ladite technique et quant à la théorie fondamentale.

«Widespread acceptance can be an important factor in ruling particular evidence admissible, and «a known technique that has been able to attract only minimal support within the community»... may properly be viewed with skepticism. ${ }^{50}$

On constate que ce dernier élément n'est plus qu'un parmi plusieurs et qu'il n'est plus nécessairement le seul facteur de validation : les tribunaux peuvent passer outre à l'évaluation par les pairs et même contester ses conclusions. L'évaluation par les pairs n'est donc plus l'unique facteur décisionnel. Bref, la Cour suprême des États-Unis refusa de déléguer aux savants des sciences dites exactes le pouvoir de décider du statut d'une science devant les tribunaux; le pouvoir décisionnel doit demeurer aux juges.

Mais il n'en demeure pas moins, à notre avis, que les juristes ne doivent accéder à des sciences étrangères qu'avec beaucoup de retenue et reconnaître, qu'en temps normal, seuls les scientifiques versés dans l'expertise à l'étude peuvent réellement juger de la valeur de la technique utilisée. C'est d'ailleurs ce qu' avait reconnu la Commission de Réforme du droit du Canada alors qu'elle avait spécifié dans un document de travail. ${ }^{51}$

«Nous pensons que les méthodes qui ne répondent pas aux critères énoncés dans l'affaire Frye devraient être mises en doute. $\gg^{52}$

$\begin{array}{ll}48 . & \text { Ibid. à la p. } 2797 . \\ 49 . & \text { Ibid. } \\ 50 . & \text { Ibid. } \\ 51 . & \text { Supra note } 39 . \\ \text { 52. } & \text { Ibid. p. } 27 .\end{array}$


D'ailleurs, la raison déterminante de la décision de la Cour suprême des États-Unis reposa d'abord et avant tout sur l'obligation des tribunaux de se soumettre à la loi : en l'instance, l'article 702 des Federal Rules of Evidence, tel qu'interprété par la Cour, en conformité avec l'économie générale de cette loi, qui en était une de libéralisation des règles relatives à la preuve, imposait une telle conclusion :

«Here, there is a specific Rule that speaks to the contested issue. Rule 702, governing expert testimony, provides:

«If scientific, technical, or other specialized knowledge will assist the trier of fact to understand the evidence or to determine a fact in issue, a witness qualified as an expert by knowledge, skill, experience, training or education, may testify thereto in the form of an opinion or otherwise. Nothing in the text of this Rule establishes «general acceptance» as an absolute prerequisite to admissibility (...). The drafting history makes no mention of Frye, and a rigid «general acceptance» requirement would be at odds with the «liberal thrust» of the Federal Rules and their «general approach of relaxing the traditional bariers to opinion testimony. (...) Given the Rules' permissive backdrop and their inclusion of a specific rule on expert testimony that does not mention «general acceptance», the assertion that the Rules somehow assimilated Frye is unconvincing. (...) That austere standard, absent from and incompatible with the Federal Rules of Evidence, should not be applied in Federal trials. ${ }^{53}$

Après avoir ainsi établi qu'il était du ressort des juges de valider la science ou la technique dont les résultats sont présentés au prétoire, le juge Blackburn, au nom de la majorité, reconnut néanmoins au pouvoir judiciaire le droit d'exclure les résultats d'une «science» ou technique jugée non valable.

Mais voilà! Alors que les tribunaux inférieurs américains sont, à notre avis, privilégiés d'avoir ainsi reçu une grille d'analyse pour effectuer le partage entre l'ivraie et le bon grain, il demeure que le magistrat à qui échoit le devoir de ce faire n'a habituellement pas les connaissances suffisantes pour effectuer ce choix, surtout s'il a à faire face à des spécialistes qui semblent convaincus de 
la valeur de leurs conclusions et de l'aberration des conclusions des experts de la partie adverse et si, dans le cadre de ce contentieux, ils se fondent sur des principes d'une science inconnue du juge, en utilisant souvent un langage hermétique, propre à cette science.

Sommes-nous tellement loin du Moyen-Âge, alors que des théologiens versés dans leur science s'arrogeaient le droit de juger la valeur de théories scientifiques tout à fait étrangères à leur art et étaient convaincus de la qualité de leur travail? Ils refusaient alors de remettre le système établi en question. Pourtant, on constate aujourd'hui les nombreuses erreurs qui découlèrent de leurs analyses et les injustices qu'ils commirent.

Puisque la quête de la vérité est le but ultime du droit de la preuve, si une réforme de ce dernier et des institutions peut améliorer la recherche de la justice et de la vérité, nous devons l'entreprendre même si elle risque de modifier substantiellement les habitudes et de diminuer les sources de revenus des avocats et des témoins experts. À ce titre, l'étude d'autres systèmes de droit peut s'avérer bénéfique.

\section{III - De la situation en droit européen continental : l'indépendance de l'expert}

La situation est fort différente en Europe continentale et, à notre avis, nous aurions intérêt à nous en inspirer.

Commençons par une présentation sommaire de la procédure pénale des pays les plus proches du Québec, tant par la langue que par la culture : la France, la Suisse et la Belgique. Il serait fastidieux d'en présenter une étude détaillée : d'autres l'ont fait avec brio. ${ }^{54}$

Nous nous attarderons ensuite au statut de l'expert judiciaire et à ses relations avec la justice.

54. Procédures pénales d'Europe, sous la direction de M. Delmas-Marty, P.U.F. 1995, 638 p.; Fennell, Phil, Criminal Justice in Europe: a Comparative Study, Oxford, Clarendon Press, 1995, 404 p.; Mueller, Gerhardg; Le Poole-Griffiths, Fr.; Edwards, John L1.J., Comparative Criminal Procedure, New York, New York University Press, 1969, 252 p. 


\section{A. La procédure pénale de l'Europe continentale (bref survol)}

Celle-ci contient à la fois des traits du système accusatoire et d'autres du système inquisitoire. La phase préliminaire du procès pénal - la constitution du dossier répressif - est de type inquisitorial, tandis que la phase du jugement est plutôt accusatoire. ${ }^{55}$

Dans une première étape, un juge d'instruction a pour tâche de constater les preuves et indices qui s'élèvent contre le suspect mais aussi les éléments qui plaident en sa faveur. C'est lui qui aura à décider s'il y a matière à procès ou non. ${ }^{56} \mathrm{Si}$ oui, l'affaire sera portée devant la cour compétente. ${ }^{57}$ Là, tout comme ici, l'accusé bénéficie de la présomption d'innocence. ${ }^{58}$ Mais un point supplémentaire distingue la procédure criminelle d'Europe de la nôtre : là, le juge a le privilège de contribuer activement à la recherche de la vérité : quoique la charge de la preuve incombe, comme chez nous, dans son intégralité, à la partie poursuivante, le juge peut suppléer aux insuffisances et soulever tout fait de nature à favoriser la manifestation de la vérité. ${ }^{59}$ Voyons maintenant les conséquences sur l'expertise.

\section{B. L'expertise}

Au niveau de l'instruction, le juge, pour décider s'il y aura poursuite ou non, peut s'adjoindre des experts. Ceux-ci ne représentent aucune partie, ils

55. H. D. Bosly, Éléments de droit de la procédure pénale, Académia-Bruylant 1996, 312 p. à la p. 10.

56. Quelques variantes existent : ainsi, en droit belge, contrairement au droit français, le juge d'instruction ne décide pas s'il y a matière à procès. Cette décision est prise par un autre juge, celui qui préside la Chambre du Conseil.

57. Entre la phase de l'instruction et celle du procès, il peut, selon les pays, y avoir d'autres étapes : ainsi, en Belgique, quand le juge d'instruction estime que son enquête est terminée, le dossier est soumis à huis clos à la Chambre du Conseil où un juge statuera sur le sort de l'instruction : soit d'arrêter les procédures, soit de porter l'affaire devant la juridiction de jugement.

58. Article $6 \$ 2$ de la Convention européenne des droits de l'homme; M. Delmas-Marty, supra note 54 pp. 470-471; 508-509; 517-522.

59. C. Hennau et J Verhaegen, Droit pénal général, Bruylant, 1991 à la p. 103. 
sont, du moins théoriquement, dégagés de toute pression extérieure. ${ }^{60}$ Ils peuvent accomplir leur tâche en toute objectivité. Cependant, en pratique, on a parfois constaté que des liens trop étroits existaient entre le parquet et les experts. En outre, tout comme chez nous au stade de l'enquête, l'absence des parties peut être source d'injustice ${ }^{61}$ : en effet, le prévenu n'a pas à cette étape, le pouvoir de contester la fiabilité des techniques utilisées, ${ }^{62}$ il pourrait donc arriver que la décision relative à la «suffisance de charges», justifiant le renvoi de l'affaire devant une juridiction de jugement, soit fondée sur une preuve scientifique erronée ou insuffisamment validée. ${ }^{63}$ En outre, lorsque l' affaire sera rendue à l'étape du procès, il se pourrait que l'indice trouvé sur les lieux de l'infraction soit disparu ou encore qu'il ait été détruit par la première analyse, ce qui pourrait rendre la contre-expertise impossible. ${ }^{64}$ Tous ces inconvénients existent d'ailleurs chez nous : le suspect n'est pas présent lors de l'enquête précédant la poursuite; ici aussi, le tout est secret et non contradictoire.

C'est donc au niveau du procès, que le droit européen est particulièrement intéressant.

60. J. Van Compernolle, La désignation, la mission et la fonction de l'expert dans L'Expertise (J. Gillardin \& P. Jadoul), Publications des Facultés universitaires Saint-Louis, Bruxelles, 1994, 105 à la p. 124.

61. M.-N. Verhaegen, «L'identification par l'analyse génétique dans le système de preuve pénale belge» dans L'Analyse génétique à des fins de preuve et les droits de l'homme, sous la direction de C. Hennau-Hublet et B M. Knoppers, Bruylant, Bruxelles, 1997, 509 p. à la p. 147.

62. Ibid. aux pp. 178 et 214 .

63. Ibid. aux pp. 184 et 214. Il semble aussi que l'on retrouve un certain lexisme des magistrats face au rapport d'expertise. Suite à un sondage adressé aux magistrats suisses. Alain Gallusser constate : «Une dernière hypothèse est que le rapport d'expertise est généralement mal compris par le magistrat et les conclusions ne sont pas toujours conformes aux attentes du magistrat. Les critiques principales adressées aux experts concernent surtout le jargon technique. Les juges ne lisent pas tout le rapport, mais uniquement la conclusion. Les juges préfèrent les conclusions catégoriques aux probabilités. Ils n'apprécient pas les conclusions trop nuancées.» Gallusser, L'indice matériel comme moyen de preuve : sa valeur et son utilisation par les magistrats, supra note 7 aux pp. 135-136.

64. Ibid. aux pp. 185 et p. 216. M.-N. Verhaegen, op. cit. note 61 à la p. 216. L. Fettweis, À propos du caractère contradictoire de l'expertise pénale, dans Jean Gillardin \& Pierre Jadoul, L'expertise, Publications des facultés universitaires Saint-Louis, Bruxelles, 1994, 96. 
Le juge peut alors s'adjoindre un ou plusieurs experts; il les choisit et leur assure, du moins en théorie, une indépendance complète. En Allemagne, le spécialiste reçoit même le qualificatif, non pas d'expert, mais «d'aide-dujuge». ${ }^{65}$ Là où plusieurs laboratoires d'expertises judiciaires existent, le juge du procès peut très bien confier le mandat à un centre distinct de celui qu'avait approché le juge d'instruction. Ainsi, à titre d'exemples, en Belgique, le juge peut recourir au Laboratoire de police judiciaire qui, évidemment, est du ressort de la sécurité publique; mais s'il veut une plus grande garantie d'objectivité, l'Institut national de criminologie et de criminalistique, qui dépend du ministère de la Justice lui est accessible; enfin, s'il le préfère, il peut avoir recours aux spécialistes des universités ou des entreprises privées. En Suisse, il y a mieux! Là, il existe de nombreux laboratoires «forensiques» mais on y trouve aussi un Institut de police scientifique et de criminologie rattaché à la Faculté de droit de l'Université de Lausanne dont l'indépendance institutionnelle assure une meilleure garantie d'objectivité.

Quoique l'État prenne les frais de l'expertise à sa charge, ils sont d'ordinaire considérés comme des frais de procédure et tomberont à la charge de l'accusé s'il est condamné. ${ }^{66}$

Évidemment, le rapport préparé par les experts nommés par le juge doit être remis aux parties et il est soumis à la contradiction des débats, car les juges ne peuvent fonder leur conviction que sur des éléments qui ont pu faire l'objet d'un débat contradictoire. ${ }^{67}$ D' ailleurs, l'exigence de la contradiction fait partie de la notion de procès équitable garantie par l'article $6 \$ 1^{\mathrm{er}}$ de la Convention européenne de sauvegarde des droits de l'homme et des libertés fondamentales, de plus, le paragraphe $6 \S 3^{\mathrm{e}}$ de la même Charte, assure à toute personne accusée

65. J. H. Langbein, «The German Advantage in Civil Procedure», (1985) 52 U. of Chi. Law Rev. 823 à la p. 835.

66. M. Franchimont, A Jacobs \& A. Masset, Manuel de procédure pénale, éd. collection scientifique de la Faculté de droit de Liège, 1989, 1157 p. aux pp. 567-68.

67. A. de Nauw, «Les règles d'exclusion relatives à la preuve en procédure pénale belge», [1990] Revue de droit pénal et de criminologie 705 à la p. 715; supra note 55 à la page 208; supra note 66 à la page 502. 
De l'expertise judiciaire dans le cadre du procès criminel et de la recherche

(1996-97) 27 R.D.U.S. de la vérité : quelques réflexions

d'une infraction, le droit d'interroger ou de faire interrroger les témoins à charge. ${ }^{68}$

En outre, dans une affaire Bönisch, ${ }^{69}$ la Cour de Strasbourg a reconnu qu'il y avait eu violation de l'article $6 \S 1$ de la Convention de sauvegarde des droits de l'homme et des libertés fondamentales car un fort déséquilibre avait existé lors du procès «en raison de la différence entre les places respectives de l'expert désigné par le tribunal et de l'expert-témoin de la défense». De plus, la Cour avait constaté en l'instance que «la défense n'avait guère (eu) de chance d'obtenir la nomination d'un contre-expert».

Cette décision devra, à notre avis, être suivie de changements importants dans la conduite des tribunaux, car il semble bien que certains juges aient parfois été portés à protéger «leur» expert et à bloquer le plaideur qui, par ses questions, aurait mis en doute les conclusions de celui-là. De plus, comme les juridictions

68. Au civil, aussi la situation peut être intéressante pour nous : par exemple, en Belgique, les parties peuvent s'entendre sur la nécessité de faire effectuer une expertise, et ce, avant même de paraître devant le tribunal. Lors de désaccord sur les conclusions de l'expert, le juge pourra s'adjoindre son propre expert. Code judiciaire belge, Procédure civile, art. 962 et ss. Feittweis, Droit judiciaire privé ( $5^{\mathrm{e}}$ éd.), Presses universitaires de Liège, 1980, $\mathrm{n}^{\mathrm{os}} 310$ à 316 bis. Le professeur Feittweis écrit au sujet du caractère contradictoire de ladite expertise : «Le caractère contradictoire de l'expertise constitue l'une de ses caractéristiques essentielles. Une expertise qui n' aurait pas été réalisée de manière contradictoire devrait être écartée (...). Diverses règles sont prévues en vue d'assurer le caractère contradictoire de l'expertise : les parties doivent être convoquées non seulement à la première réunion mais aussi à toutes les opérations d'expertise, à moins que les parties n'aient dispensé l'expert de les en informer. L'expert doit demander aux parties de prendre position sur ce point lors de la première réunion (...) les experts doivent attendre les parties et faciliter leur conciliation (a. 972 al. 3) (...). En toute hypothèse, les parties doivent être entendues contradictoirement, c'est-à-dire en présence l'une de l'autre (...). Les experts ont l'obligation de faciliter la conciliation des parties (a. 972 al. 3) (...) L'article 980 vise le cas où plusieurs experts ont été nommés. En ce cas, leur travail est collégial et leur rapport doit être leur oeuvre commune. En pareille hypothèse, il est généralement désigné un expert rapporteur, chargé de procéder à certaines recherches matérielles préliminaires, de réunir la documentation, de préparer le projet des préliminaires puis celui de la seconde partie du rapport. L'expert qui exprime un avis différent ne doit pas nécessairement garder l'anonymat; il peut en prendre la responsabilité. En toute hypothèse, en cas de divergence d'avis, le juge peut décider de les entendre en audience (art. 987) et, à ce moment, chaque expert devra défendre son propre point de vue.» Voir : Feittweis, Droit judiciaire privé, aux $\mathrm{n}^{\text {os }} 312,316$ et $316 \mathrm{~B}$.

69. C.E.D.H. arrêt Bönisch c/Autriche, 6 mai 1985 série A, N $^{\circ} 92$ SS 33 et 34. Voir : A. Kohl, Implication de l'article 6, alinéa $1^{\text {er }}$ C.E.D.H. en procédure pénale, J.T. 1988 p. 435 No 8. 
de fond statuent essentiellement sur des dossiers préétablis par les juges d'instruction, certains allèguent que l'inculpé se trouve parfois démuni face à la conviction du juge déjà acquise sur la culpabilité. ${ }^{70}$ Enfin, on souligne que ce droit de contestation ne peut effectivement être exercé, lorsque l'objet de l'expertise a disparu ou que, à la suite des manipulations, il a subi de profondes modifications, ${ }^{71}$ ce qui peut parfois être le cas, entre autres, lors d'analyse d'A.D.N.

Bref, en Europe tout comme ici, on doit accorder à la défense une égalité des armes en matière d'expertise, sinon les droits fondamentaux de la défense à un procès équitable pourront être baffoués. ${ }^{72}$

Il est symptomatique de constater qu'alors que notre système contradictoire est fort contesté quant au statut de l'expertise, ${ }^{73}$ les collègues européens semblent plutôt satisfaits de leur système : ainsi, en France, la Commission justice pénale et droits de l'homme, présidée par la professeure Mireille Delmas-Marty, après avoir suggéré plusieurs réformes à la procédure pénale, jugea fort satisfaisant le système d'experts nommés par la Cour, ${ }^{74}$ en Italie $^{75}$ et au Portugal,${ }^{76}$ lors de réformes substantielles qui incorporèrent à leur droit plusieurs éléments propres à notre système contradictoire, le législateur maintint néanmoins le système d'experts nommés par la Cour.

70. A.L. Feittweis, La charge de preuve en droit pénal belge et la présomption d'innocence, dans Les droits de la défense en matière pénale, Liège, Éd. du Jeune Barreau, 1985, pp. 134157.

71. Supra note 61 à la p. 216; L. Fettweis, À propos du caractère contradictoire de l'expertise pénale, op. cit. note 64 .

72. F. Golgüklü, Le procès équitable et l'administration des preuves dans la jurisprudence de la Cour européenne des droits de l'homme, Mélanges offerts à Jacques Velu, Bruylant, 1992, p. 1365.

73. Voir entre autres écrits : supra note 11; supra note 26; «Forensic Science and Expert Evidence», (1994) 21 Journal of Law and Society 136; supra note 65; L. Fettweis, supra note 71 ; J. D. Havard, supra note 5.

74. Commission justice pénale et droits de l'homme, La Mise en état des affaires pénales, (1990) voir à la p. 197.

75. Nuovo Codice di Procedura Penale, (1988) a. 220.

76. Código de Processo Penal, (1987) a. 158. 
De l'expertise judiciaire dans le cadre du procès criminel et de la recherche de la vérité : quelques réflexions

Que pouvons-nous tirer de cette courte présentation du rôle de l'expert en droit continental européen? Y aurait-il des leçons à tirer de l'expérience de ces pays pour améliorer notre droit. Peut-être, mais à la condition, selon nous, de jouxter aux pratiques de l'Europe continentale, les garanties de notre système contradictoire. Voyons donc...»

\section{IV- Quelques suggestions pour assurer une meilleure objectivité des experts et une efficience accrue.}

Force est de constater que le spécialiste choisi par le juge est libéré des pressions provenant des parties : le risque que son objectivité soit affectée par son désir de voir «sa partie» gagner «sa cause» y est absent. Sa tâche n' y est que la recherche de la vérité, sans se soucier des résultats. ${ }^{77}$ Néanmoins, nous l'avons vu, le droit étudié nous semble lacunaire quant à l'expertise au niveau de l'instruction : puisque, à ce niveau, la procédure est essentiellement écrite, secrète, et non contradictoire, l'absence des parties peut être source d'injustice. Les Pays-Bas ont, semble-t-il, trouvé la solution à cette lacune : le juge d'instruction doit aviser par écrit le prévenu de l'endroit et de l'heure où l'expertise sera effectuée. Ce dernier, ou son avocat, ainsi qu'un expert de son choix, pourront y être présents. De plus, dans les quinze jours qui suivent la remise du rapport d'expert, le prévenu peut demander au juge d'instruction de désigner un autre expert pour effectuer une contre-expertise. ${ }^{78}$

Ensuite, le pouvoir de contester les conclusions de l'expert nous semble essentiel à la recherche de la vérité. La France assure ce droit depuis l'adoption du Code de procédure pénale de 1960 : quatre possibilités sont offertes à ce titre :

- L'article 165 dudit code permet aux parties de demander à la Cour d'enjoindre à l'expert du tribunal d'effectuer des analyses spécifiques supplémentaires. S'il y a refus, le requérant peut porter appel. ${ }^{79}$

77. Supra note 11 à la p. 211; supra note 65.

78. Supra note 61 à la p. 216.

79. Une garantie similaire au niveau de l'instruction est accordée en Belgique depuis l'adoption, le 10 juillet 1997, de la Loi relative à l'amélioration de la procédure pénale au stade de l'information et de l'instruction. (Voir l'art. 14) [ $3^{\mathrm{e}}$ session, $49^{\mathrm{e}}$ législature]. 
- Lors de la réception du rapport d'expertise, les parties sont appelées à le commenter. Elles peuvent alors demander d'autres expertises ou même la nomination d'un autre expert. Il y a possibilité d'appel lors d'un refus.

- Lors du procès, l'article 168 prévoit la présence de l'expert et l'obligation de justifier ses conclusions. Alors le président peut lui transmettre les questions posées par les procureurs; il peut aussi permettre que les questions lui soient directement adressées.

- $\quad$ Enfin, la défense peut présenter à la barre son propre expert, qui pourra offrir une contre-expertise.

Selon le doyen Spencer, cette dernière alternative est cependant peu utilisée car :

«In the first place, legal aid is not available to pay for private expert... Secondly, a private expert, unlike a contre-expert appointed by the juge d'instruction, does not have access to the clues and exhibits; thus defendants will usually try to challenge the expertise by pressing for a contre-expertise and calling a private expert is a course of last ressort..»

En fait, on devrait toujours permettre à la défense l'accès aux indices et aux détails concernant l'expertise.

À ce sujet, le professeur Alldridge écrivait fort justement :

«If no effective monitoring takes place, or if the defence does not have a full description of the manner in which the tests are carried out, it does not really matter what provisions are made for the exclusion of evidence.. ${ }^{81}$

Il nous semble que, chez nous, le compromis suivant améliorerait grandement la situation, tout en gardant l'essentiel du système contradictoire et,

80. Supra note 11 à la p. 232.

81. P. Alldridge, «Recognizing Novel Scientific Techniques: DNA as a test case», (1992) Crim. L. Rev. 686 à la p. 698. 
en plus, en maintenant le pouvoir décisionnel du juge : la Cour devrait être assistée de son propre expert qui aurait la tâche de rédiger un rapport d'expertise.

Évidemment, au criminel, le juge est habituellement saisi de la cause trop tard pour que son expert puisse effectuer la première analyse : celle-ci fut déjà faite dans le cadre de l'enquête, avant même que des poursuites ne soient initiées. Néanmoins, si le juge est assisté d'un expert de son choix, ce dernier analysera pour lui le premier rapport remis au procureur de la Couronne. Et, en outre, puisque parfois l'expert du juge aura à analyser de nouveau les éléments de preuve, les corps policiers auront tout intérêt à conserver les exhibits, les pièces à conviction, pour assurer la possibilité de cette expertise; à défaut d'avoir eu une telle prudence, leur cause pourrait avorter...!

Chaque partie pourrait continuer à être assistée d'un expert, si elle le désire, pour l'analyse du rapport de l'expert du juge et dans la préparation des questions à lui poser. La confrontation demeurerait; de celle-ci, le juge aurait une meilleure compréhension des problèmes, il pourrait lui-même questionner son expert après avoir pu bien délimiter les sujets à controverse.

Mentionnons néanmoins que, selon les données américaines, le contreinterrogatoire des experts demeure exceptionnel, premièrement parce que la majorité des cas est réglée au moyen de la négociation des plaidoyers (plea bargaining) et que, deuxièmement, ce qui nous semble symptomatique, la défense ne dispose ordinairement pas des moyens de contester les lettres de créance de l'expert et les méthodes employées. ${ }^{82}$

Évidemment, le spécialiste choisi par le tribunal aurait une préséance découlant du fait que son statut assurerait son objectivité; mais ce statut ne serait pas nécessairement garant de la qualité de son travail et l'assurance d'un contreinterrogatoire devrait demeurer. On pourrait, cependant, envisager la possibilité d'adresser les questions au juge qui les transmettrait à l'expert, évitant ainsi l'agressivité caractéristique des contre-interrogatoires traditionnels.

82. J. L. Peterson \& J. E. Murdock, Forensic Science Ethics : Developing an Integrated System of Support and Enforcement, (1989) 34 J. Forensic Sci. 749 à la p. 750. 
Enfin, il est évident que le grand danger d'un système où l'expert est nommé par le juge réside dans le fait que le scientifique peut avoir trop de pouvoir et détenir de facto le pouvoir décisionnel; mais ici, il s'agit plus d'une question ayant trait au statut du juge et à son professionnalisme : il doit demeurer le maître de la décision et s'il abdique son rôle, il le fera autant face aux experts des parties que face à l'expert de la Cour.

Donc, le juge serait assisté d'un expert de la Cour, sa décision serait peutêtre plus éclairée grâce aux lumières de ce spécialiste qui aurait des compétences techniques et scientifiques que le magistrat ne possède pas; mais encore faut-il que cet auxiliaire de la justice ait oeuvré dans des conditions qui assurent son impartialité, ce qui nous amène au point suivant.

\section{V - De l'indépendance institutionnelle des centres d'expertise en criminalistique appliquée}

Nous venons de le voir, pour que l'expert se consacre à la recherche de la vérité objective de façon la plus impartiale possible, il importe qu'il soit dégagé de toute pression provenant des parties, qu'elle soit financière ou psychologique. À cet égard, il est, en outre, nécessaire qu'il ne soit pas influencé indûment par son employeur et qu'il ne soit pas soumis à des pressions institutionnelles. Il doit bénéficier de la liberté scientifique la plus large possible.

Or, il appert que, presque partout, les experts en criminalistique appliquée oeuvrent dans le cadre de laboratoires d'État sous le contrôle, soit de corps policiers (pensons au F.B.I. National Academy à Quantico, Virginie, ou encore au Laboratoire de la Gendarmerie royale du Canada, à Ottawa), soit d'un ministère responsable de la sécurité publique (telles la Sous-Direction de la police technique et scientifique, située à Ecully, France, qui relève du ministère de l'Intérieur, section Direction générale de la police nationale, ou encore le Laboratoire de sciences judiciaires et de médecine légale, qui relève du ministère de la Sécurité publique du Québec). La direction de ce dernier allègue qu'il jouit d'un statut autonome; mais aucun texte légal (loi ou règlement) ne l'assure. Ce laboratoire est donc soumis, quoiqu'on puisse dire, aux directives finales du ministère de la Sécurité Publique du Québec et, dans une situation exceptionnelle, il ne dispose d'aucune protection formelle contre 
une possible ingérence. Dans un même ordre d'idées Peterson et al. ${ }^{83}$ ont publié les résultats d'un sondage qui révèle que $80 \%$ des laboratoires de police scientifique («forensic science laboratories») d'Amérique du Nord étaient intégrés aux forces policières ou à des bureaux des procureurs de la poursuite. Il y a plus : $30 \%$ des membres desdits laboratoires étaient des officiers de police. On comprend que $57 \%$ des laboratoires qui ont répondu au sondage de ces auteurs aient rapporté qu'ils refusaient d'effectuer des analyses pour la défense.

Or, connaissant le caractère souvent déterminant au procès du témoignage de l'expert «forensique», n'est-il pas inquiétant de constater le peu d'autonomie des centres où se font les analyses? Les autorités ne peuvent-elles pas imposer leur philosophie à leurs employés? Quid du chercheur dont les conclusions seraient trop souvent favorables à la défense? Et que dire de celui qui mettrait en doute les fondements mêmes d'une technique jusque-là trouvée infaillible et utilisée à grande échelle par les corps policiers, reçue par les tribunaux sans questionnement? Ne pourrait-il pas être discipliné ou à tout le moins réduit au silence?

En outre, il ne faut pas oublier qu'il y a place pour la subjectivité et l'erreur, même lors d'utilisation de techniques jugées par plusieurs quasi infaillibles et que, en conséquence, la mentalité des experts en sciences judiciaires ainsi que la philosophie des centres où ils oeuvrent peuvent parfois avoir certaines conséquences sur leurs conclusions.

\section{A. L'exemple de la preuve d'empreintes digitales}

Rendons notre démonstration exigeante : il eut été facile de prouver la possibilité d'erreur causée par la subjectivité dans l'emploi de techniques peu exactes (telles que l'identification visuelle de la voix, ou celle de la signature). Non! Confrontons nos constatations à une technique jugée très fiable et très concluante par les tribunaux : la preuve dactyloscopique (c'est-à-dire celle d'empreintes digitales).

83. J.L. Peterson, S. Mihajlovic et L. Bedrosian, «The Capabilities, Uses and Effects of the National's Criminalistics Laboratories», (1985) 30 J. Forensic Sci. 10-23. 
Dans certains pays, une identification formelle n'est établie que si le nombre minimal de minuties correspondantes (c.-à-d. points de similitude) a pu être mis en évidence; ainsi, au Danemark, le nombre minimal de points sera de 10; de 16 en Grande-Bretagne. Christophe Champod constate cette disparité et les conséquences qui en découlent :

«Cela signifie paradoxalement qu' une empreinte présentant 12 minuties en Grande-Bretagne n'a aucune valeur identificatrice effective, alors que dans la majorité des autres pays, cette empreinte sera formellement identifiée et la preuve introduite dans les tribunaux. Le cas de la France est encore plus étonnant puisque la Prefecture de Paris se fie à la valeur de 17 minuties, alors que la police nationale s'attache à 12 points! Ainsi, un suspect arrêté en province sur la base d'une trace digitale avec 12 minuties pourrait être relaxé à Paris.» ${ }^{84}$

Or, il appert que les États-Unis et le Canada ne requièrent pas un nombre minimum de points et laissent la démonstration de l'identité, et la détermination de la valeur probante de celle-ci, à l'appréciation de l'expert.

Evett et Williams sont deux spécialistes en la matière au Home Office Forensic Science Service à Londres, centre de renommée internationale. Voici quelques extraits d'un de leurs écrits relatifs à la norme britannique qui laissent songeur :

«Probably because of the sixteen points standard, a practice has grown in the U.K. service which the team did not find in the other countries visited. A fingerprint expert will generally reach an inner conviction about the correctness of an identification long before he has found 16 points. His or her subsequent activity will center on establishing that features which are clearly visible in the print can also be seen in the poorer quality mark. The print is used as a guide for scrutinizing the mark. This is called in some quarters «teasing the points out.» ${ }^{85}$

84. C. Champod, Reconnaissance automatique et analyse statistique des minuties sur les empreintes digitales, Université de Lausanne, Faculté de droit, 1996.

85. I.W. Evett \& R.L. Williams, «A Review of the Sixteen Points Fingerprint Standard in England and Wales», (1996) 46 J. Forensic Ident. 49 à la p. 68. 
D'ailleurs, ces auteurs, spécialistes en la matière, indiquent clairement que la dactyloscopie n'est pas une science exacte :

«... the determination of the individual points is subjective and the collaborative study showed that experts vary widely in their judgements of individual points. ${ }^{86}$

Nous sommes d'autant plus songeur, lorsque nous apprenons qu'au Canada aucune norme relative au nombre minimal de minuties n'existe. ${ }^{87}$

Evett et Williams ${ }^{88}$ avaient envoyé les mêmes empreintes digitales pour fins d' analyse et d'identification à plusieurs laboratoires d'expertises judiciaires renommés. Utilisant les systèmes de recherche automatique d'identification les plus sophistiqués, dont le AFIS (Automatic Fingerprint Identification System), les chercheurs de ces laboratoires eurent à détecter les points spécifiques à chaque empreinte que sont les bifurcations ou les arrêts des lignes papillaires appelées aussi minuties et à les comparer à l'échantillon témoin. Nous avons déjà mentionné que le nombre minimum de points exigés par la justice pour que

86. Ibid. à la p. 65. Certains autres opinent d'ailleurs dans le même sens à l'effet que le standard numérique favorise la détection de minuties imaginaires. G.D. Tiller, «Fingerprint Identification in United Kingdom - Do they really need 16 points?», (1983) 6 Can. Ident. Soc. Newsletter, p. 5 à 8.

87. «Historiquement, sous l'influence de Locard (1914) en particulier, la règle quantitative des «douze points» a été admise par une majorité d'États... Dans la réalité, les circonstances de temps, de fait, de lieu, ramènent souvent la population à des dimensions locales et limitent d'autant les probabilités, tout en renforçant la valeur d'identification qu'elles représentent. L'affirmation formelle de l'identité peut donc se faire, en pratique, sur la base d'empreintes qui n'ont qu'un nombre limité de minuties, parfois même 6,7 ou 8, comme cela est admis dans certains pays (Inde, Afrique du Sud) ou par l'Association internationale pour l'identification. À la suite de cette résolution, les États-Unis et le Canada ne requièrent plus un nombre minimum de points et laissent la démonstration de l'identité et la détermination de la valeur probante de celle-ci à l'appréciation de l'expert dactyloscopique. Il est rare qu'une identification soit obtenue avec moins de 7 à 8 points dans ces pays, bien que cela soit théoriquement possible. Ici, l'éthique de l'expert est importante. Un contrôle de qualité ainsi qu'une confirmation d'une identification par un expert indépendant paraissent essentiels pour garantir que l'identification est justifiée dans ces cas limites.» [Nos italiques]. P. A. Margot et C. J. Lennard, Les méthodes de détection des empreintes digitales, Institut de police scientifique et de criminologie, Université de Lausanne $\left(6^{\mathrm{e}}\right.$ éd. révisée) 1994,182 p. aux pp. 8-9.

88. Supra note 85. 
l'empreinte puisse servir en preuve, varie selon les pays : 16 en Grande-Bretagne et en Italie, 12 en France (17 à Paris), 12 en Suisse et 10 au Danemark... ${ }^{89}$ Les réponses furent fort variables : quelques laboratoires trouvèrent 8 points de convergence, d'autres, jusqu'à 40. Comme quoi, la présumée infaillibilité de l'identification par empreinte digitale dépend de l'interprétation du technicien et de la valeur de l'appareillage. Elle est donc évidemment... faillible! Donc, lorsque les juristes écrivent qu'une identification positive par empreintes digitales est concluante, il serait de mise d'ajouter, à tout le moins, lors du bon fonctionnement d'un appareillage de qualité et en faisant abstraction de l'erreur humaine possible chez le technicien-analyste.

Alors, ne peut-on pas déduire qu'un expert, oeuvrant dans le contexte policier, sous supervision policière, préparé a priori par les enquêteurs et informé du dossier, pourrait «étendre son objectivité pour la teinter de subjectivité» $?^{90}$ Et que dire du fait que la preuve par profil génétique, parfois encore plus déterminante pour une déclaration de culpabilité que celle d'empreintes digitales suit une grille d'analyse analogue à cette dernière? ${ }^{91}$

\section{B. L'indépendance institutionnelle}

L'indépendance institutionnelle nous semble donc essentielle à l'impartialité des chercheurs. L'analogie avec l'exigence de l'indépendance institutionnelle du pouvoir judiciaire n'est pas sans intérêt : puisque les conclusions de l'expert sont souvent déterminantes au procès, son autonomie doit être protégée. une unité de doctrine dans l'évaluation de leur force probante», Mélanges en l'honneur du professeur Jean Gauthier, (1996) Revue pénale suisse, à la p. 233; C. Champod, Reconnaissance automatique et analyse statistique des minuties sur les empreintes digitales, Thèse de doctorat, Institut de police scientifique et de criminologie de l'Université de Lausanne, 1996, pp. 2-3.

90. Supra note 10 à la p. 725 : «Knowing that a defendant has confessed to the rape muder of a child, how, lawmen wonder, can they be satisfied that the interpretation of the laboratory findings is not in some way colored by that knowledge? Some insist that the scientist should know nothing of the background of an investigation to preclude such possibility.»

91. P. A. Margot et C. Champod, supra note 89 à la p. 229. 
Le statut de l'Institut de police scientifique et de criminologie de l'Université de Lausanne nous semble répondre à cette exigence d'autonomie : composante de l'Université de Lausanne, rattaché organiquement à la Faculté de droit, il profite de la liberté académique propre aux universités : tant dans la recherche fondamentale qu'appliquée ou dans l'enseignement des sciences «forensique», la liberté scientifique y est protégée.

Si le but de l'expertise est la recherche de la vérité et non le succès de l'inculpation, ne devrait-on pas accorder à chaque centre de criminalistique appliquée une indépendance institutionnelle assurée? Et puisque la recherche doit y être une composante importante, pourquoi ne pas incorporer ces centres à des universités pour garantir la multidisciplinarité? À l'Institut de police scientifique et de criminologie de Lausanne, les facultés de droit, de sciences et de médecine participent, évidemment; mais, on profite aussi de l'impact et de la présence des facultés de sciences sociales (sociologie, psychologie, deviance, statistiques), des Hautes études commerciales (H.E.C.) (informatique) de théologie (éthique) et même de lettres (mathématiques, philosophie). ${ }^{92}$

Un statut intermédiaire est accordé au Forensic Science Service et au Metropolitan Police Forensic Science Laboratory britanniques..$^{93}$ Ils sont, depuis 1991, des agences autonomes gouvernementales et peuvent accepter de faire des analyses à la demande soit de la poursuite, soit de la défense. Il semble, cependant, que le changement ait eu des conséquences plus théoriques que pratiques, que la défense ait eu peu accès auxdits services (d'ailleurs, là où il n'y aurait qu'un seul centre de criminalistique appliquée bien outillé, ce qui est le cas du Québec, on comprend que la police serait la première à lui confier un mandat ce qui, en pratique, en bloquerait l'accès à la défense); enfin, il semble, en outre, que les scientifiques à l'emploi de ces services, soient peu portés à mettre en doute les conclusions de collègues ${ }^{94}$ du même service. Donc, la présence de plus d'un laboratoire bien équipé semble nécessaire pour assurer à la défense l'accès à des services de qualité égale à ceux offerts à la poursuite.

92. P. A. Margot et C. J. Lennard, IPSC, Lausanne, 1993.

93. Nous avons déjà mentionné que le Laboratoire de sciences judiciaires et de médecine légale du Ministère de la sécurité publique du Québec est dans une situation à peu près analogue.

94. P. Alldridge, «Forensic Science and Expert Evidence», (1994) 21 Journal of Law and Society, 136 à la p. 139. 
Enfin, l'expert n'aidera à la découverte de la vérité que si sa compétence est reconnue et que s'il oeuvre dans un centre dont la qualité est maintenue.

\section{VI- De la création de comités d'agrément ou d'accréditation}

Nous avons vu que c'est lorsqu'apparaissent au prétoire les résultats de l'utilisation de nouvelles techniques inusitées, ou de sciences innovatrices, que les tribunaux se trouvent les plus démunis. À qui se fier pour savoir, premièrement, si le principe fondamental sur lequel repose la technique ou la science est valide et, deuxièmement, pour connaître la valeur des appareils ou des méthodes utilisées? Devrait-il y avoir dans chaque pays un comité d'accréditation qui les validerait?

Comme la plupart des principes fondamentaux des sciences judiciaires sont identiques dans le monde entier, il nous semble qu'il serait préférable que toute nouvelle découverte soit soumise à un centre international d'agrément. Dès 1929, d'ailleurs, une Académie internationale de criminalistique avait vu le jour, dont le siège était à Vienne. ${ }^{95}$ On y prévoyait, à l'article 2 que «L'Académie internationale combattra l'empirisme dans le domaine de la criminalistique et de la technique policière» et, à l'article 37, la possibilité d'instituer une commission de surexperts pour analyser la valeur de certaines techniques. Malheureusement la seconde guerre mondiale sonna le glas de cette académie.

Actuellement il arrive que certains gouvernements confient à des groupes de savants de leur pays l'étude de la valeur de certaines techniques pour, ensuite, aviser le pouvoir judiciaire de leurs conclusions; ainsi, pour ne nommer que celles-là, l'analyse spectographique (identification visuelle de la voix) ${ }^{96}$ et la technique d'identification par A.D.N. ${ }^{97}$ firent l'objet de telles analyses. Mais pour éviter le danger d'influences indues ainsi que des lacunes certaines dans des pays n'ayant pas assez d'experts indépendants versés en la matière, ne serait-il pas préférable de confier une telle étude à un comité international? Au niveau des influences indues, il ne faudrait certes pas sous-estimer celles provenant des

95. Statuts de l'Académie internationale de criminalistique, (1929) 1 Revue internationale de criminalistique 85 .

96. National Academy of Science, On the Theory and Practice of Voice Identification (1979).

97. National Academy of Science, The Evaluation of Forensic D.N.A. Evidence, (23 mai 1997). 
concepteurs des appareils et des fabricants de ceux-ci qui ont des intérêts économiques ou professionnels à ce qu'ils soient validés. Or, comme dans plusieurs pays, les scientifiques concernés sont peu nombreux et forment une confrérie assez hermétique, il y a danger de favoritisme.

Enfin, ledit Comité international pourrait être appelé à agréer les laboratoires pour assurer une constance dans la qualité de ces derniers; la question de l'accréditation des laboratoires de criminalistique appliquée refait d'ailleurs surface dès qu'une nouvelle technique apparaît ${ }^{98}$ ou dès qu'une mauvaise analyse des données ou une malpratique est imputée à un de ces laboratoires ou un de ses experts et qu'il appert qu'elles furent la cause de condamnations injustifiées.

\section{Conclusion}

Nous constatons que la plupart des erreurs judiciaires qui résultèrent soit de la négligence ou, pire, soit de la malhonnêteté d'experts en sciences «forensiques», le furent au détriment de l'accusé. De nombreuses personnes eurent donc à porter le poids de longues années d'emprisonnement ou, à tout le moins, d'accusations qui s'avérèrent non fondées à cause d'erreurs judiciaires résultant de preuves scientifiques inexactes ou même parfois contrefaites.

Il est à la mode, fort heureusement, d'écrire sur les droits fondamentaux de l'accusé; peut-on oublier que son droit le plus fondamental est d'abord et avant tout celui à la vérité?

Le droit à une défense pleine et entière est enchâssé à l'article 7 de la Charte canadienne des droits et libertés, le droit à la présomption d'innocence s'y trouve aussi, ainsi qu'à l'article 11(d). Pour que ces droits soient effectifs, il importe d'abord et avant tout que le procès assure la découverte de la vérité

98. Ainsi pour l'accréditation des laboratoires effectuant des analyses d'A.D.N. : voir L. Viau, La problématique de la preuve d'A.D.N. en droit pénal : La recherche du point d'équilibre entre vérité scientifique et vérité du droit dans C. Hennau-Hublet \& B.M. Knoppers, L'analyse génétique à des fins de preuve et les droits de l'homme, Bruylant, Bruxelles, 1997, 509 p. à la p. 276. 
et que, s'il y a condamnation, celle-ci ne soit fondée que sur une preuve contrôlée par un juge des faits apte à le faire, parfois parce qu' assisté lorsque besoin est.

Or, il nous semble que notre système contradictoire (adversarial) soit inapproprié lorsqu'il y a présence de l'expertise et qu'une réforme soit aussi nécessaire au niveau des institutions de criminalistique appliquée.

Se pourrait-il que les forces conjuguées de l'inertie et de l'appât du gain bloquent ces réformes qui nous semblent aller dans le sens des intérêts supérieurs de la justice? Espérons que, si le système demeure inaltéré, ce sera parce que d'autres motifs, que nous n'avons pas découverts, en auront imposé le maintien. 